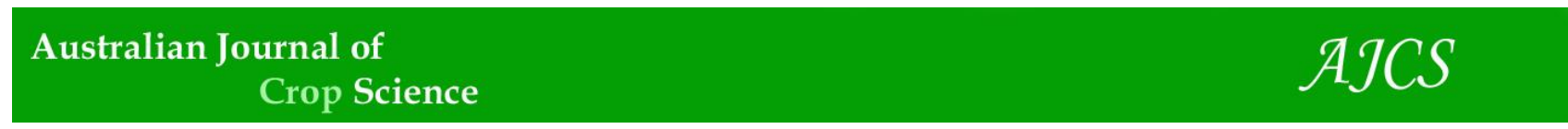

AJCS 10(7):956-963 (2016)

ISSN:1835-2707

DOI: $10.21475 /$ ajcs.2016.10.07.p7616

\title{
Combining ability analysis of cassava (Manihot esculenta Crantz) genotypes for cassava mosaic disease in Zambia
}

\author{
Patrick Chiza Chikoti ${ }^{*}$, Paul Shanahan ${ }^{2}$, Rob Melis ${ }^{2}$ \\ ${ }^{1}$ Zambia Agriculture Research Institute, Mount Makulu Research Station, Private bag 7 Chilanga, Zambia \\ ${ }^{2}$ University of KwaZulu-Natal, African Centre for Crop improvement, P/Bag X01, Scottsville, South Africa
}

*Corresponding author: chizachikoti@hotmail.com

\begin{abstract}
Despite the importance of cassava as a staple crop in Luapula province and other regions of Zambia, there is a lack of information on the resistance to diseases and associated combining ability of the locally cultivated cassava cultivars. Therefore, this study was carried out in 2010/2011 to: identify progeny with resistance to cassava mosaic disease (CMD); evaluate the performance of $F_{1}$ progeny for agronomic traits and determine general combining ability and specific combining ability for resistance to CMD. A total of 809 genotypes comprising of parents and progeny were developed through controlled crossing and evaluated using $\alpha$-lattice design. The general combining ability (GCA) and SCA mean squares (MS) were highly significant ( $<<0.001)$ for CMD. The total sum of squares (SS) for CMD was mainly accounted for by specific combining ability (SCA) effects (67.9\%). Bangweulu a local highly susceptible cultivar had the most negative, significant $(\mathrm{P}<0.001)$ GCA effect among the landraces. In summary, the results indicated that the local landraces (Bangweulu and Kampolombo) used as parents can be sources of CMD resistance.
\end{abstract}

Keywords: Cassava mosaic disease; progeny; general combining ability; specific combining ability.

\section{Introduction}

Pests and diseases are among the most important biotic constraints to cassava production. Cassava mosaic disease (CMD) is a devastating disease in Africa and yield losses may be as high as $100 \%$ (Thresh et al., 1994). In Zambia yield losses to CMD of between 50 to $70 \%$ have been recorded in farmers' fields (Muimba-Kankolongo et al., 1997). The disease is transmitted by the whitefly vector (Bemisia tabaci Gennadius) from plant to plant within the field (Calvert and Thresh, 2002) and also spread from one field to another through infected cuttings (Legg et al., 2011). Yield reduction due to CMD varies from agroecological zone to zone and so do the management strategies. The strategies, which are not mutually exclusive, include: phytosanitation, cultural methods (cultivar mixtures) (Thresh and Cooter, 2005), vector management through use of insecticides (Calvert and Thresh, 2002) and resistance breeding (Jennings and Iglesias, 2002). For longer term cost-effective management, breeding for resistance is essential. In view of yield reduction caused by CMD, plant breeding has the potential of creating new progenies to sustainably manage the disease.

To produce new genetic combinations and generate genetic information, mating designs have been used in breeding cassava, among them the diallel (designs I, II, and III) and factorial or North Carolina (designs I, II, and III). The information generated from these designs is used to determine the general combining ability (GCA) and specific combining ability (SCA) of the parents. In this study, the NCII mating design was used to generate progeny from crosses involving a set of male and female parents. The NCII mating design has been used in several crop species, for example, maize (Eberhart and Gardner, 1966), sugarcane (Hogarth et al., 1981), pearl millet (Angarawai et al., 2008), and wheat (Virk et al., 1985), Kamau et al. (2010) have also used the design to study the inheritance of yield ability and secondary traits in cassava.

Generation of genetic information involves managing a large number of genotypes, particularly the progeny of crosses. Therefore, selection (of plant type and reaction to certain diseases) during the seedling trial and clonal evaluation trial was previously done visually without data recording (Ceballos et al., 2004). In addition, no replication was made during early selection. In this study, to overcome some of the shortcomings of previous strategies, crosses were separated into four replications and each cross randomly allocated to the four replications. The study was conducted to: i) identify parents and progeny with resistance to CMD ii) evaluate the performance of $F_{1}$ progeny for agronomic traits and iii) determine general combing and specific combing ability for resistance to CMD.

Results

Performance of the $F_{1}$ crosses

The CMD scores ranged from 1.09 (Bangweulu x TMS3001) to 1.55 (Chikula $\mathrm{x}$ TMS190), respectively. Mean fresh root yield ranged from 0.51 (Bangweulu $\mathrm{x}$ Mweru) to $0.74 \mathrm{~kg}$ plant $^{-1}$ (Bangweulu x TME2). The majority of the clones had developed storage roots, however, clones in some crosses had none. Mean harvest index ranged from a low of 0.51 (Chikula $\mathrm{x}$ TMS190 and Chila $\mathrm{x}$ Nalumino) to a high of 0.59 (Kampolombo x Nalumino). Plant height across the families varied from 69.32 (Bangweulu x TMS190) to $85.94 \mathrm{~cm}$ (Chila $\mathrm{x}$ Nalumino). The lowest mean fresh biomass of 0.90 
$\mathrm{kg}$ plant $^{-1}$ was recorded in crosses Chikula $\mathrm{x}$ TME2 and Chikula $\mathrm{x}$ Nalumino and the highest of $1.26 \mathrm{~kg}_{\text {plant }}{ }^{-1}$ in Bangweulu x Mweru. Mean leaf retention ranged from 2.03 (Chila $\mathrm{x}$ Mweru) to 2.39 (Bangweulu $\mathrm{x}$ TME2). The lowest mean root number of 4.46 was recorded in family Bangweulu $\mathrm{x}$ Nalumino and the highest of 6.22 in Bangweulu x TME2. Root size ranged from 3.15 (Chikula x TMS190) to 3.73 (Kampolombo x TMS190).

\section{Combining ability mean squares for cassava mosaic disease} and agronomic traits

The CMD general combining ability (GCA) and specific combining ability mean squares (MS) were highly significant $(\mathrm{P}<0.001)$ (Table 1). The GCA SS for male parents accounted for less of the CMD crosses sums of squares (SS) at $12.5 \%$ than the GCA SS for female parents at $19.6 \%$. The SCA SS accounted for $67.9 \%$ of the CMD crosses SS. The GCA MS for the fresh root yield for the female parents were highly significant $(\mathrm{P}<0.001)$, while the GCA MS for male parents and the SCA MS were not. The GCA effects for harvest index for the female parents were significant $(\mathrm{P}<0.05)$. The SCA MS were highly significant for plant height $(\mathrm{P}<0.001)$ and non-significant for fresh root yield, harvest index, total biomass and root size. The GCA SS \% (male and female) was higher than the SCA SS \% for fresh root yield $(70.2 \%)$, total biomass $(69.7 \%)$ and root size $(60.3 \%)$. The GCA:SCA ratio for fresh root yield (2.36), total biomass (2.30) and root size (1.52) was higher than one. For cassava mosaic disease, harvest index (0.63) and plant height (0.45), GCA:SCA ratio was lower than one.

\section{General combining ability effects}

In the female parents, Bangweulu had the most significant $(\mathrm{P}<0.001)$ negative GCA effect for CMD (Table 2). Kampolombo also had a significant $(\mathrm{P}<0.01)$ negative GCA effect. Positive significant GCA effects were recorded in Chikula $(\mathrm{P}<0.001)$ and Chila $(\mathrm{P}<0.01)$. In the male parents, significant $(\mathrm{P}<0.01)$ negative GCA effects were recorded for TME2 and Nalumino. TMS190 had a significant $(\mathrm{P}<0.001)$ positive GCA effect. The GCA effect for fresh root yield for Bangweulu was positive and significant $(\mathrm{P}<0.01)$ while Chikula had a significant $(\mathrm{P}<0.001)$ negative GCA effect. For plant height, Nalumino had a significant $(\mathrm{P}<0.01)$ positive GCA effect (Table 3). Chikula had a significant $(\mathrm{P}<0.01)$ GCA effect for harvest index (Table 4). Kampolombo also had a significant $(\mathrm{P}<0.01)$ positive GCA effect for root size while Chikula had a significant $(\mathrm{P}<0.01)$ negative effect.

\section{Specific combining ability effects}

Eleven crosses had significant SCA effects for CMD, five of which had negative effects, namely: Bangweulu x TMS190 $(\mathrm{P}<0.001)$, Bangweulu $\mathrm{x}$ TMS3001 $(\mathrm{P}<0.001)$, Chikula $\mathrm{x}$ Mweru $(\mathrm{P}<0.001)$, Chila $\mathrm{x}$ Nalumino $(\mathrm{P}<0.001)$ and Kampolombo $x$ Nalumino $(\mathrm{P}<0.01)$, and the other six had significant positive effects, namely: Chikula x TMS190 ( $\mathrm{P}<0.01)$, Kampolombo x TMS190 $(\mathrm{P}<0.01)$, Chila $\mathrm{x}$ TMS $3001(\mathrm{P}<0.001)$, Bangweulu x Mweru $(\mathrm{P}<0.001)$, Bangweulu $\mathrm{x}$ Nalumino $(\mathrm{P}<0.001)$ and Chikula $\mathrm{x}$ Nalumino $(\mathrm{P}<0.01)$ (Table 5). Bangweulu $\mathrm{x}$ TME2 had significant $(\mathrm{P}<0.01)$ positive effects for plant height (Table 6). Significant negative effects were recorded in Bangweulu x TMS190 $(\mathrm{P}<0.001)$, Mweru x Chila $(\mathrm{P}<0.01)$ and Chikula $\mathrm{x}$ TME2 $(\mathrm{P}<0.01)$.

\section{Phenotypic correlations between traits}

Most of the traits were significantly correlated with one another (Table 7). However, there was no significant correlation between CMD and fresh root yield, harvest index, total biomass, leaf retention, root number and root size. Similarly no correlation was observed between leaf retention and fresh root yield. A significant $(\mathrm{P}<0.001)$ and high positive correlation was recorded between total biomass and fresh root yield. Significant $(\mathrm{P}<0.001)$ positive correlations were recorded between: harvest index and fresh root yield. Positive and significant $(\mathrm{P}<0.001)$ correlation were also recorded between plant height and fresh root yield. Root number and fresh root yield; harvest index and total biomass; leaf retention and root number were also positive and significantly $(\mathrm{P}<0.001)$ correlated. Positive and significant correlation was also recorded between plant height and total biomass, leaf retention, root number and root size. Total biomass was also significantly $(\mathrm{P}<0.001)$ positively correlated with root number and root size. Leaf retention was also significantly $(\mathrm{P}<0.001)$ positively correlated with root size.

\section{Trait contribution to genotype performance}

Most of the total variation (74.5\%) was accounted for by the first three principal components (PCs) (Table 8). The PC1 accounted for $41.4 \%$ of the total variation with an eigenvalue of 2.49. The traits that contributed the most to the PC1 were harvest index, biomass, fresh root yield and root size. The PC2 accounted for $17.7 \%$ of the total variation with harvest index, plant height and leaf retention being the major contributors. The PC 3 accounted for $15.4 \%$ of the total variation with harvest index, leaf retention and root size as the main contributors.

\section{Estimates of heterosis}

The best five crosses with desired negative CMD heterosis (relative to mid-parent value) were Bangweulu x TMS3001 ($113.9 \%)$, Chikula $\mathrm{x}$ TMS3001 $(-113.9 \%)$, Chikula $\mathrm{x}$ Nalumino (-107.9\%), Bangweulu x Nalumino (-107.8\%), Chikula x TMS190 (-104.6\%) (Table 9). All these crosses involved a resistant and susceptible parent. The best five crosses with positive heterosis for plant height were Kampolombo $x$ TMS190 (2589.5\%), Kampolombo $x$ TMS3001 (2547.5\%), Kampolombo x Mweru (1782.0\%), Bangweulu x TME2 (1691.5\%), and Kampolombo x TME2 $(1518.5 \%)$. For total biomass the best five crosses with positive heterosis were Chikula $\mathrm{x}$ Nalumino (21.9\%), Kampolombo x TMS3001 (18.4\%), Kampolombo x TME2 (16.8\%), Bangweulu x TME2 (15.4\%) and Kampolombo x TMS190 (11.4\%). For fresh root yield most of the crosses had positive heterosis and the best five were Bangweulu $\mathrm{x}$ TME2 (24.2\%), Bangweulu x TMS3001 (20.9\%), Kampolombo x Nalumino (19.1\%), Kampolombo x TMS3001 (17.1\%) and Bangweulu x Mweru (16.6\%). Harvest index also recorded positive heterosis in most of the crosses. The best five crosses with positive heterosis for harvest index were Bangweulu $x$ TME2 (11.5\%), Kampolombo x Nalumino (10.4\%), Chikula x Nalumino (9.2\%), Bangweulu x TMS3001 (8.4\%), and Cikula x TME2 (8.3\%). 
Table 1. Cross means for cassava mosaic disease, fresh root yield, harvest index, plant height, total biomass, leaf retention, root number and root size at the clonal evaluation stage, Mansa, 2011

\begin{tabular}{|c|c|c|c|c|c|c|c|}
\hline \multirow[b]{2}{*}{ Source } & \multicolumn{7}{|c|}{ Mean square value } \\
\hline & Df & CMD & FRY & $\mathrm{HI}$ & $\mathrm{PH}$ & TB & $\mathrm{RS}$ \\
\hline Rep & 3 & 0.282 & 0.3676 & 0.00613 & 596.15 & 0.955 & 0.437 \\
\hline Crosses & 19 & $0.0711^{* * *}$ & $0.0219 \mathrm{~ns}$ & $0.00214 *$ & $82.38^{* * * *}$ & $0.0391 \mathrm{~ns}$ & $0.113 \mathrm{~ns}$ \\
\hline GCA (Male) & 4 & $0.042 * * *$ & $0.0141 \mathrm{~ns}$ & $0.000449 \mathrm{~ns}$ & $77.46^{*}$ & $0.0457 \mathrm{~ns}$ & $0.107 \mathrm{~ns}$ \\
\hline GCA (Female) & 3 & $0.088^{* * *}$ & $0.0783^{* * * *}$ & $0.004677^{*}$ & $58.82 \mathrm{~ns}$ & $0.1117^{*}$ & $0.289 *$ \\
\hline SCA & 12 & $0.076^{* * *}$ & $0.0103 \mathrm{~ns}$ & $0.002076 \mathrm{~ns}$ & $89.91^{* *}$ & $0.0188 \mathrm{~ns}$ & $0.0711 \mathrm{~ns}$ \\
\hline Error & 57 & 0.00604 & 0.01288 & 0.001118 & 28.78 & 0.0295 & 0.0748 \\
\hline \multicolumn{8}{|l|}{ Crosses SS } \\
\hline GCA (Male) & & 12.5 & 13.6 & 4.4 & 19.8 & 24.6 & 19.9 \\
\hline GCA (Female) & & 19.6 & 56.6 & 34.4 & 11.3 & 45.1 & 40.4 \\
\hline SCA & & 67.9 & 29.8 & 61.2 & 68.9 & 30.3 & 39.7 \\
\hline GCA:SCA ratio & & 0.47 & 2.36 & 0.63 & 0.45 & 2.3 & 1.52 \\
\hline
\end{tabular}

CMD (Cassava mosaic disease); FRY (fresh root yield, kg plant ${ }^{-1}$ ); HI (harvest index); PH (plant height, cm); TB (total biomass, kg plant ${ }^{-1}$ ); LR (leaf retention); RN (root number); RS (root size); GCA (general combining ability); SCA (specific combining ability); SS (sums of squares); ******* Significant at $\mathrm{P}<0.5, \mathrm{P}<0.01$ and $\mathrm{P}<0.001$ probability, respectively.

Table 2. General combining ability effects for cassava mosaic disease and fresh root yield of nine cassava parents

\begin{tabular}{lllllll}
\hline & \multicolumn{2}{l}{ Cassava mosaic disease scores (1 to 5) } & \multicolumn{3}{l}{ Fresh root yield $\left(\mathrm{kg} \mathrm{plant}^{-1}\right)$} \\
\cline { 2 - 7 } Genotype & Mean & GCA & GCA (SE) & Mean & GCA & GCA (SE) \\
\hline Bangweulu & 1.23 & $-0.07^{* * * *}$ & 0.02 & 0.69 & $0.06^{* * *}$ & 0.03 \\
Chikula & 1.37 & $0.06^{* * *}$ & 0.02 & 0.56 & $-0.08^{* * *}$ & 0.03 \\
Chila & 1.36 & $0.05^{* *}$ & 0.02 & 0.62 & -0.02 & 0.03 \\
Kampolombo & 1.27 & $-0.04^{* *}$ & 0.02 & 0.68 & 0.04 & 0.03 \\
TMS190 & 1.39 & $0.08^{* * *}$ & 0.02 & 0.65 & 0.01 & 0.03 \\
TMS3001 & 1.33 & 0.02 & 0.02 & 0.65 & 0.02 & 0.03 \\
Mweru & 1.30 & -0.01 & 0.02 & 0.67 & 0.03 & 0.03 \\
Nalumino & 1.26 & $-0.05^{* *}$ & 0.02 & 0.59 & -0.05 & 0.03 \\
TME2 & 1.28 & $-0.04^{* *}$ & 0.02 & 0.63 & -0.01 & 0.03 \\
\hline
\end{tabular}

GCA (general combining ability); SE (standard error); *, **, *** significant at $\mathrm{P}<0.05, \mathrm{P}<0.01, \mathrm{P}<0.001$

Table 3. General combining ability effects for plant height and total biomass.

\begin{tabular}{|c|c|c|c|c|c|c|}
\hline \multirow[b]{2}{*}{ Genotype } & \multicolumn{3}{|c|}{ Plant height $(\mathrm{cm})$} & \multicolumn{3}{|c|}{ Total biomass $\left(\mathrm{kg} \mathrm{plant}^{-1}\right)$} \\
\hline & Mean & GCA & GCA (SE) & Mean & GCA & GCA (SE) \\
\hline Bangweulu & 78.9 & -0.50 & 1.20 & 1.12 & 0.062 & 0.04 \\
\hline Chikula & 77.3 & -2.15 & 1.20 & 0.97 & $-0.094 * *$ & 0.04 \\
\hline Chila & 80.3 & 0.87 & 1.20 & 1.04 & -0.026 & 0.04 \\
\hline Kampolombo & 81.2 & 1.78 & 1.20 & 1.12 & 0.058 & 0.04 \\
\hline TMS190 & 77.9 & -1.54 & 1.34 & 1.11 & 0.048 & 0.04 \\
\hline TMS3001 & 79.7 & 0.30 & 1.34 & 1.06 & -0.003 & 0.04 \\
\hline Mweru & 78.4 & -1.03 & 1.34 & 1.12 & 0.059 & 0.04 \\
\hline Nalumino & 83.1 & $3.71 * *$ & 1.34 & 0.10 & -0.063 & 0.04 \\
\hline TME2 & 78.0 & -1.44 & 1.34 & 1.02 & -0.041 & 0.04 \\
\hline
\end{tabular}


Table 4. General combining ability effects for harvest index and root size.

\begin{tabular}{|c|c|c|c|c|c|c|}
\hline \multirow[b]{2}{*}{ Genotype } & \multicolumn{3}{|c|}{ Harvest index } & \multicolumn{3}{|c|}{ Root size } \\
\hline & Mean & GCA & GCA (SE) & Mean & GCA & GCA (SE) \\
\hline Bangweulu & 0.56 & 0.0118 & 0.01 & 3.49 & 0.080 & 0.06 \\
\hline Chikula & 0.53 & $-0.0181 * *$ & 0.01 & 3.27 & $-0.128 * *$ & 0.06 \\
\hline Chila & 0.53 & -0.0072 & 0.01 & 3.33 & -0.075 & 0.06 \\
\hline Kampolombo & 0.56 & 0.0135 & 0.01 & 3.52 & $0.122 * *$ & 0.06 \\
\hline TMS190 & 0.54 & 0.0040 & 0.01 & 3.39 & -0.011 & 0.07 \\
\hline TMS3001 & 0.55 & 0.0071 & 0.01 & 3.49 & 0.085 & 0.07 \\
\hline Mweru & 0.54 & -0.0023 & 0.01 & 3.46 & 0.065 & 0.07 \\
\hline Nalumino & 0.54 & -0.0042 & 0.01 & 3.39 & -0.018 & 0.07 \\
\hline TME2 & 0.54 & -0.0047 & 0.01 & 3.28 & -0.121 & 0.07 \\
\hline
\end{tabular}

GCA (general combining ability); SE (standard error); *, **, *** significant at $\mathrm{P}<0.05, \mathrm{P}<0.01, \mathrm{P}<0.001$.

Table 5. Mean performance and specific combining ability effects for cassava mosaic disease scores of 20 crosses.

\begin{tabular}{lccc}
\hline & & \multicolumn{2}{c}{ Cassava mosaic disease score } \\
\cline { 2 - 3 } Cross & Mean & SCA effects & SCA(SE) \\
\hline Bangweulu xTMS190 & 1.17 & $-0.14^{* * *}$ & 0.04 \\
Chikula x TMS190 & 1.55 & $0.10^{* *}$ & 0.04 \\
Chila x TMS190 & 1.37 & -0.07 & 0.04 \\
Kampolombo x TMS190 & 1.47 & $0.12^{* *}$ & 0.04 \\
Bangweulu x TMS3001 & 1.09 & $-0.17^{* * *}$ & 0.04 \\
Chikula x TMS3001 & 1.41 & 0.02 & 0.04 \\
Chila x TMS3001 & 1.51 & $0.12^{* * *}$ & 0.04 \\
Kampolombo x TMS3001 & 1.32 & 0.02 & 0.04 \\
Bangweulu x Mweru & 1.37 & $0.14^{* * *}$ & 0.04 \\
Chikula x Mweru & 1.19 & $-0.17^{* * *}$ & 0.04 \\
Mweru x Chila & 1.42 & 0.06 & 0.04 \\
Kampolombo x Mweru & 1.23 & -0.03 & 0.04 \\
Bangweulu x Nalumino & 1.36 & $0.17^{* * *}$ & 0.04 \\
Chikula x Nalumino & 1.40 & $0.08^{* *}$ & 0.04 \\
Chila x Nalumino & 1.14 & $-0.18^{* * *}$ & 0.04 \\
Kampolombo x Nalumino & 1.14 & $-0.08^{* *}$ & 0.04 \\
Bangweulu x TME2 & 1.19 & -0.01 & 0.04 \\
Chikula x TME2 & 1.31 & -0.02 & 0.04 \\
Chila x TME2 & 1.39 & 0.06 & 0.04 \\
Kampolombo x TME2 & 1.21 & -0.02 & 0.04 \\
\hline Mean & 1.31 & & \\
\hline SCA
\end{tabular}

SCA (specific combing ability); SE (standard error); *, **, *** significant at $\mathrm{P}<0.05, \mathrm{P}<0.01, \mathrm{P}<0.001$, respectively.

Table 6. Mean performance and specific combining ability effects for plant height of 20 crosses.

\begin{tabular}{|c|c|c|c|}
\hline \multirow[b]{2}{*}{ Cross } & \multicolumn{2}{|c|}{ Plant height $(\mathrm{cm})$} & \multirow[b]{2}{*}{ SCA(SE) } \\
\hline & Mean & SCA & \\
\hline Bangweulu xTMS190 & 69.32 & $-8.07 * * *$ & 2.81 \\
\hline Chikula x TMS190 & 79.07 & 0.34 & 2.81 \\
\hline Chila x TMS190 & 81.87 & 3.11 & 2.81 \\
\hline Kampolombo x TMS190 & 84.31 & 4.63 & 2.81 \\
\hline Bangweulu x TMS3001 & 78.17 & -1.06 & 2.81 \\
\hline Chikula x TMS3001 & 7716 & -0.41 & 2.81 \\
\hline Chila x TMS3001 & 82.02 & 1.42 & 2.81 \\
\hline Kampolombo x TMS3001 & 81.56 & 0.05 & 2.81 \\
\hline Bangweulu x Mweru & 80.82 & 2.91 & 2.81 \\
\hline Chikula x Mweru & 77.97 & 1.72 & 2.81 \\
\hline Mweru x Chila & 73.27 & $-6.00 * *$ & 2.81 \\
\hline Kampolombo x Mweru & 81.54 & 1.36 & 2.81 \\
\hline Bangweulu x Nalumino & 82.29 & -0.36 & 2.81 \\
\hline Chikula x Nalumino & 84.83 & 3.84 & 2.81 \\
\hline Chila x Nalumino & 85.86 & 1.85 & 2.81 \\
\hline Kampolombo x Nalumino & 79.60 & -5.33 & 2.81 \\
\hline Bangweulu x TME2 & 84.08 & $6.58 * *$ & 2.81 \\
\hline Chikula x TME2 & 70.35 & $-5.49 * *$ & 2.81 \\
\hline Chila x TME2 & 78.48 & -0.38 & 2.81 \\
\hline Kampolombo x TME2 & 79.07 & -0.71 & 2.81 \\
\hline Mean & 79.43 & & \\
\hline
\end{tabular}


Table 7. Phenotypic correlation coefficients for CMD and agronomic traits for 800 genotypes at the clonal evaluation stage, 2011.

\begin{tabular}{|c|c|c|c|c|c|c|c|c|}
\hline CMD & - & & & & & & & \\
\hline FRY & 0.199 & - & & & & & & \\
\hline HI & 0.039 & $0.624 * * *$ & - & & & & & \\
\hline LR & 0.055 & 0.218 & 0.007 & - & & & & \\
\hline TB & 0.268 & $0.947 * * *$ & $0.436 * * *$ & $0.318 * *$ & - & & & \\
\hline $\mathrm{PH}$ & 0.240 & $0.551 * * *$ & 0.118 & $0.427 * * *$ & $0.680 * * *$ & - & & \\
\hline RN & 0.133 & $0.819 * * *$ & $0.463 * * *$ & 0.160 & $0.771 * * *$ & $0.352 * *$ & - & \\
\hline \multirow[t]{2}{*}{$\mathrm{RS}$} & 0.112 & $0.522 * * *$ & $0.300 * *$ & $0.294 * *$ & $0.553 * * *$ & $0.581 * * *$ & $0.296 * *$ & - \\
\hline & CMD & FRY & $\mathrm{HI}$ & $\mathrm{PH}$ & TB & LR & RN & $\mathrm{RS}$ \\
\hline
\end{tabular}
CMD (Cassava mosaic disease); FRY (fresh root yield, $\left.\mathrm{kg} \mathrm{plant}^{-1}\right) ; \mathrm{HI}$ (harvest index); PH (plant height, $\mathrm{cm}$ ); TB (total biomass, $\mathrm{kg}_{\text {plant }}{ }^{-1}$ ); LR (Leaf retention);
RN (root number); RS (root size); DMC (dry matter content) $*, * * * * *$ Significantly different from zero at the $0.05,0.1$ and 0.01 probability levels, respectively (two-tailed test)

Table 8. Principal component coefficients of the various traits with loadings of the various yield and yield components.

\begin{tabular}{lllll}
\hline Traits & PC1 & PC2 & PC3 & PC4 \\
\hline HI & 0.372 & -0.413 & 0.187 & 0.356 \\
PH & 0.054 & 0.790 & 0.538 & 0.229 \\
Bio & 0.572 & 0.045 & 0.026 & 0.053 \\
FRY & 0.607 & -0.079 & 0.068 & 0.141 \\
LR & 0.213 & 0.429 & -0.817 & 0.185 \\
RS & 0.343 & 0.120 & 0.056 & -0.874 \\
Eigenvalue & 2.485 & 1.06 & 0.924 & 0.811 \\
Total variation \% & 41.4 & 17.7 & 15.4 & 13.5 \\
\hline
\end{tabular}

PC (principal component); $\mathrm{PH}$ (plant height, cm); HI (harvest index); TB (total biomass, $\mathrm{kg} \mathrm{plant}^{-1}$ ); FRY (fresh root yield, kg plant ${ }^{-1}$ ); LR (Leaf retention); RS (root size).

Table 9. Mean performance and mid-parent heterosis (\%) for traits evaluated at the clonal evaluation stage, Mansa, 2011.

\begin{tabular}{|c|c|c|c|c|c|c|c|c|c|c|c|c|c|c|}
\hline Parents & CMD & & $\mathrm{PH}$ & & $\mathrm{BIO}$ & & FRY & & $\mathrm{HI}$ & & LR & & $\mathrm{RS}$ & \\
\hline crosses & Mean & MPH & Mean & $\mathrm{MPH}$ & Mean & MPH & Mean & MPH & Mean & MPH & Mean & MPH & Mean & $\mathrm{MPH}$ \\
\hline P1 & 3.23 & & 83.8 & & 1.01 & & 0.54 & & 0.46 & & 2.43 & & 3.38 & \\
\hline P2 & 2.86 & & 68.0 & & 0.88 & & 0.45 & & 0.45 & & 2.55 & & 3.15 & \\
\hline P3 & 1.67 & & 96.0 & & 1.37 & & 0.75 & & 0.53 & & 2.00 & & 3.32 & \\
\hline P4 & 1.67 & & 61.0 & & 1.06 & & 0.62 & & 0.56 & & 3.63 & & 3.74 & \\
\hline P5 & 1.20 & & 55.8 & & 1.16 & & 0.63 & & 0.52 & & 2.87 & & 4.40 & \\
\hline P6 & 1.23 & & 51.2 & & 0.90 & & 0.48 & & 0.51 & & 2.30 & & 4.00 & \\
\hline P7 & 1.67 & & 74.8 & & 1.07 & & 0.61 & & 0.53 & & 2.43 & & 3.20 & \\
\hline P8 & 1.33 & & 67.8 & & 0.76 & & 0.39 & & 0.43 & & 2.53 & & 3.00 & \\
\hline P9 & 1.27 & & 61.5 & & 0.97 & & 0.47 & & 0.45 & & 2.57 & & 3.55 & \\
\hline 1 & 1.17 & -104.6 & 69.3 & -51.0 & 1.16 & 7.65 & 0.71 & 13.15 & 0.56 & 7.60 & 2.28 & -37.00 & 3.38 & -65.75 \\
\hline 2 & 1.55 & -48.6 & 76.1 & 1416.5 & 0.94 & -8.15 & 0.51 & -2.90 & 0.51 & 3.00 & 2.21 & -49.95 & 3.15 & -58.75 \\
\hline 3 & 1.37 & -6.5 & 81.9 & 596.5 & 1.12 & -14.05 & 0.66 & -2.65 & 0.55 & 2.80 & 2.03 & -40.25 & 3.32 & -37.70 \\
\hline 4 & 1.47 & 3.4 & 84.3 & 2589.5 & 1.22 & 11.40 & 0.71 & 8.35 & 0.56 & 2.20 & 2.24 & -100.60 & 3.74 & -79.45 \\
\hline 5 & 1.09 & -113.9 & 78.2 & 1067.0 & 1.09 & 0.25 & 0.72 & 20.85 & 0.57 & 8.35 & 2.27 & -9.35 & 3.61 & -22.35 \\
\hline 6 & 1.41 & -63.5 & 77.2 & 1757.5 & 0.98 & 8.60 & 0.56 & 9.10 & 0.52 & 4.55 & 2.16 & -26.50 & 3.28 & -25.85 \\
\hline 7 & 1.51 & 6.1 & 82.0 & 843.5 & 1.01 & -12.20 & 0.62 & 0.05 & 0.54 & 2.45 & 2.26 & 10.60 & 3.47 & -2.60 \\
\hline 8 & 1.32 & -13.0 & 81.6 & 2547.5 & 1.16 & 18.35 & 0.72 & 17.05 & 0.57 & 3.35 & 2.35 & -61.55 & 3.61 & -72.15 \\
\hline 9 & 1.37 & -107.9 & 80.8 & 149.0 & 1.26 & 21.90 & 0.74 & 16.60 & 0.55 & 5.95 & 2.17 & -26.40 & 3.68 & 24.95 \\
\hline 10 & 1.19 & -107.8 & 78.0 & 655.5 & 1.05 & 6.80 & 0.62 & 8.15 & 0.52 & 3.15 & 2.12 & -37.75 & 3.48 & 34.75 \\
\hline 11 & 1.42 & -24.9 & 73.3 & -1214.5 & 1.08 & -14.30 & 0.65 & -3.20 & 0.55 & 2.05 & 2.03 & -18.35 & 3.21 & 10.50 \\
\hline 12 & 1.23 & -43.8 & 81.5 & 1362.5 & 1.10 & 3.15 & 0.66 & 4.10 & 0.53 & -0.95 & 2.14 & -89.00 & 3.52 & -41.15 \\
\hline 13 & 1.36 & -92.1 & 82.3 & 646.0 & 0.95 & 6.10 & 0.56 & 9.05 & 0.52 & 7.55 & 2.18 & -29.90 & 3.50 & 16.95 \\
\hline 14 & 1.40 & -70.1 & 84.8 & 1691.5 & 0.98 & 15.40 & 0.55 & 13.00 & 0.53 & 9.15 & 2.26 & -28.65 & 3.29 & 25.05 \\
\hline 15 & 1.14 & -36.5 & 85.9 & 394.5 & 0.99 & -7.00 & 0.56 & -1.55 & 0.51 & 2.75 & 2.20 & -6.75 & 3.30 & 29.80 \\
\hline 16 & 1.14 & -36.2 & 79.6 & 1518.5 & 1.08 & 16.75 & 0.70 & 19.05 & 0.60 & 10.35 & 2.29 & -79.70 & 3.47 & -36.15 \\
\hline 17 & 1.19 & -105.7 & 84.1 & 1141.5 & 1.17 & 17.75 & 0.74 & 24.15 & 0.57 & 11.45 & 2.39 & -10.60 & 3.27 & -34.15 \\
\hline 18 & 1.32 & -75.0 & 70.4 & 560.0 & 0.90 & -2.95 & 0.55 & 8.50 & 0.53 & 8.25 & 2.15 & -40.85 & 3.21 & -9.95 \\
\hline 19 & 1.39 & -7.9 & 78.5 & -27.0 & 0.97 & -19.35 & 0.60 & -0.65 & 0.52 & 3.45 & 2.25 & -3.65 & 3.36 & 8.60 \\
\hline 20 & 1.21 & -25.8 & 79.1 & 1782.0 & 1.05 & 3.40 & 0.61 & 7.05 & 0.52 & 2.05 & 2.28 & -81.60 & 3.31 & -80.45 \\
\hline
\end{tabular}

CMD (cassava mosaic disease); PH (plant height, $\mathrm{cm}$ ); BIO (biomass, kg plant ${ }^{-1}$ ); FRY (fresh root yield, kg plant ${ }^{-1}$ ); HI (harvest index); LR (leaf retention); RS (root size); MPH (mid-parent heterosis) P1(Bangweulu); P2 (Chikula); P3 (Chila); P4 (Kampolombo); 1 (Bangweulu x TMS190); 2 (Chikula x TMS190); 3 (Chila x TMS190); 4

(Kampolombo x TMS190); 5 (Bangweulu x TMS3001); 6 (Chikula x TMS3001); 7 (Chila x TMS3001); 8 (Kampolombo x TMS3001); 9 (Bangweulu x Mweru); 10

(Chikula x Mweru); 11 (Chila x Mweru); 12 (Kampolombo x Mweru); 13 (Bangweulu x Nalumino); 14 (Chikula x Nalumino); 15 (Chila x Nalumino); 16 (Kampolombo x

Nalumino); 17 (Bangweulu x TME2); 18 (Chikula x TME2); 19 (Chila x TME2); 20 (Kampolombo x Mweru). 


\section{Discussion}

Progeny with increased resistance to CMD were produced from crosses between the selected parents. For the individual clones, the full range of CMD scores from 1 to 5 with a mean of 1.31 was recorded in the $800 \mathrm{~F}_{1}$ progeny. Significant differences between the $F_{1}$ progenies and parents were observed for CMD resistance. Low mean CMD scores were recorded in crosses of Bangweulu x TMS3001, Bangweulu x TMS190 and Chila $x$ Nalumino indicating high tolerance to CMD. The overall cross mean, for fresh root yield was 0.64 $\mathrm{kg} \mathrm{plant}^{-1}\left(6.4 \mathrm{t} \mathrm{ha}^{-1}\right)$ at 7 MAP. This apparently low yield may be explained by the early harvesting at 7 MAP. The mean yield is comparable to that recorded by Munga (2008), Mtunda (2010) and Kamua (2010). It has been documented (Ngeve, 1999) that cassava undergoes root bulking from 4 MAP and during the initial stages root growth is slow (Hahn et al., 1979). For individual progeny plants, fresh root yield ranged from $0.10 \mathrm{~kg}$ plant ${ }^{-1}\left(1.0 \mathrm{t} \mathrm{ha}^{-1}\right)$ to $3.35 \mathrm{~kg}$ plant $^{-1}(33.5$ $\left.\mathrm{t} \mathrm{ha}^{-1}\right)$, while for the parents, it ranged from $0.1 \mathrm{~kg} \mathrm{plant}^{-1}(1.0$ $\left.\mathrm{t} \mathrm{ha}^{-1}\right)$ to $1.9 \mathrm{~kg}^{-1 a n t^{-1}}\left(19 \mathrm{t} \mathrm{ha}^{-1}\right)$ with a mean of $0.56 \mathrm{~kg}$ plant $^{-1}$. This is indicative of the progress being made in developing high yielding, early bulking cassava genotypes. Cassava cultivars currently cultivated in Zambia are usually harvested 2 to 3 years after planting. Understandably, early bulking is one of the traits desired by farmers. The PCA helped to explain the relative contribution of the various traits to genotype performance and as expected fresh root yield made the greatest contribution.

Genetic information was generated at the clonal evaluation stage in order to estimate general combining abilities or breeding values of the parental lines for the traits of interest (Ceballos et al., 2004). The GCA and SCA MS were highly significant implying that additive and non-additive gene effects were both important. For CMD, $67.9 \%$ of the variation was explained by SCA indicating that non-additive gene action predominated over additive gene action for this trait. Kamua (2010) also reported higher SCA effects for CMD. However, Lokko et al. (2006) reported the reverse. The significant GCA and SCA MS recorded for the traits of interest indicate sufficient genetic variance in the population to enable effective selection for the traits (Ragsdale and Smith, 2007). However, the breeding strategy adopted will depend on the prevalent gene action. The significance of parents' MS for CMD was indicative of the diversity of the parents suggesting that both landraces and introductions could be sources of resistance to CMD. Significant negative SCA effects were recorded for five crosses for CMD, namely: Bangweulu x TMS190, Bangweulu x TMS3001, Chikula x Mweru, Chila x Nalumino and Kampolombo x Nalumino. A cross with significant and negative SCA signifies that the cross is more resistant than mean performance of the rest of the crosses based on the expectation of additive effects. A cross with positive SCA means that the cross was more susceptible than the mean performance based on the expectation of additive effects. For most of the crosses negative mid-parent heterosis was observed for CMD emphasizing the progress made in breeding for resistance.

The GCA SS comprised $70.2 \%$ of the crosses SS indicated the strong influence of additive gene action in the expression of fresh root yield. The GCA and SCA MS for plant height were also significant meaning that both additive and nonadditive gene action was important in the determination of this trait. However, since the SCA SS comprised $68.9 \%$ of crosses SS, non-additive gene action predominated over additive gene action for this trait. Bangweulu had a significant positive GCA effect for fresh root yield implying that this landrace which was used as a female parent made an above average contribution to increased fresh root yield in all of its crosses. TMS190 also a significant positive GCA effect for total biomass and therefore made an above average contribution to increased biomass accumulation in all of its crosses. Similarly for Kampolombo, which made a significant above average positive contribution to root size in all of its crosses. Bangweulu had the most significant negative GCA effect for CMD which is an indication of its important contribution to CMD resistance. Kampolombo also had a significant negative GCA effect for CMD. The significant negative GCA effects for CMD exhibited by these two parents implicates with the role of additive gene action in determining improved resistance to CMD in the crosses involving these two parents. These parents were consequently considered to be good general combiners for improved resistance to CMD. Chikula, TMS190 and Chila exhibited significant positive effects for CMD suggesting that the three parents determined increased susceptibility of their progeny relative to the mean performance of all progeny. The GCA MS for fresh root yield was significant; however, the SCA MS was not. This implies that additive gene action was predominant over non-additive gene action for this trait. Bangweulu x TME2 had significant positive SCA effects for plant height meaning that non-additive gene action was important for the trait. Bangweulu recorded a significant negative GCA effect for CMD and a high positive GCA effect for fresh root yield suggesting that it would be a valuable parent for high yield in association with high CMD resistance, Based on its significant positive GCA effect for total biomass, TMS190 may be categorised as a good general combiner for this trait. In the same vein, Nalumino was a good general combiner for plant height. By way of contrast, Chikula had significant large negative GCA effects for biomass and root size which indicated that it was a poor general combiner for these two traits.

Despite grafting the $\mathrm{F}_{1}$ cassava progenies with $\mathrm{CMD}$ infected scions, it is interesting to note that CMD score was not significantly correlated with any of the other traits. This is probably due to low virus titre as the clones were virus free when planted and harvested early (7 MAP). The nonsignificant correlation suggests the need for caution in the selection of progeny for improved resistance to CMD in association with improved performance in the other traits. Zacarias (2008) also recorded non-significant correlations between CBSD and yield. Fresh root yield was positively correlated $(\mathrm{P}<0.001)$ with harvest index, root size, root number, leaf retention and biomass. The significant and high positive correlation between fresh root yield and harvest index has been reported by other workers (Cach et al., 2006; Kawano, 2003; Kawano et al., 1998). The relevance of significant correlations between harvest index and other traits is that harvest index can be used as a selection criterion.

\section{Materials and methods}

\section{Site description}

The study was carried out during the 2010/11 rainy season at Mansa research station, Luapula Province, Zambia. The research station is located in agroecological zone III and receives more than $1000 \mathrm{~mm}$ of rainfall per annum. 


\section{Trial layout and management}

Cuttings were taken from the middle part of each selected plant in the seedling trial to establish the clonal evaluation trial. The cuttings were planted on 10 December 2010 in a $4 \mathrm{x}$ $5 \alpha$-lattice design with four replications. The genotypes from each cross were separated into groups. Each of the $20 \mathrm{~F}_{1}$ crosses comprising 40 progeny was divided equally across the four replications. Each sibling within a cross was represented by a single plant in a plot. Planting was done at a standard $1 \times 1 \mathrm{~m}$ inter and intra-row spacing providing a plant population of 10000 plants $\mathrm{ha}^{-1}$. The replications were separated by $2 \mathrm{~m}$ wide alleys. Planting was done on ridges (farm practice of planting cassava in Luapula Province). Weeding of the trial was done as necessary and no fertiliser was applied.

\section{Virus inoculation technique}

Although Mansa has high CMD prevalence, the whitefly population is too low to ensure efficient and rapid virus transmission. To breed for CMD resistance, it is important to consider the infection method and ensure an even distribution of the viruses. Therefore, grafting was employed to transmit the viruses to the test plants in addition to planting the diseased spreaders in all the four replications. Grafting was done at 3 months after planting (MAP) by cutting the scions of the test plants in a tapered fashion and the rootstocks of the infected plants in a wedge. The scion and the rootstock were then held firmly together by wrapping a strip of plastic around the graft union.

\section{Data collection}

Plants were scored for CMD at 5,6, and 7 MAP using a scale of 1-5 (Hahn et al., 1980) where: 1, no symptoms observed; 2 , mild chlorotic pattern over entire leaflets or mild distortion at the base of leaflets only, with the remainder of the leaflets appearing green and healthy; 3, moderate mosaic pattern throughout the leaf, narrowing and distortion of the lower one-third of leaflets; 4, severe mosaic, distortion of two thirds of the leaflets and general reduction of leaf size; 5, severe mosaic distortion of the entire leaf. Plants were harvested by hand at 7 MAP for yield and yield components. The number and fresh mass $(\mathrm{kg})$ of all the storage roots (fresh root yield) per plant were counted and recorded. Root size was categorized into three classes: small, below $3 \mathrm{~cm}$ root diameter at widest point; medium, $5-7 \mathrm{~cm}$ root diameter at widest point; and large, $7 \mathrm{~cm}$ or wider root diameter at widest point. Harvest index was determined as a percentage of fresh root mass relative to total fresh biomass. Leaf retention was assessed on a 1-5 scale (Lenis et al., 2006), where: 1, very poor retention; 2 , less than average retention; 3 , average leaf retention; 4 , better than average retention; and 5, outstanding leaf retention.

\section{Data analysis}

The residual maximum likelihood procedure (REML) in Genstat version 14 statistical package was used to analyse the data. The relative contributions of the various traits was carried out based on Jollife's (2002) approach using principal component analysis (PCA) procedure in Genstat. Mid-parent heterosis (relative to mid-parent value) was determined for all the variables. The performance of the genotypes within each of the crosses was expressed on a cross mean basis for the various traits. The general combining ability (GCA), effects and specific combining ability (SCA) effects (genetic components) were estimated from the expected mean squares. The mean squares of GCA and SCA were used to determine GCA:SCA ratios (Haussmann et al., 1999). The parental cultivars and progeny were regarded as fixed effects while the replications were considered as random effects. Therefore, inferences drawn from this study cannot be generalised and extended to other populations. The GCA and SCA effects were estimated using the following model (Hallauer and Miranda, 1988): $\mathrm{Y}_{\mathrm{ijk}}=\mu+\mathrm{Fg}_{\mathrm{i}}+\mathrm{Mg}_{\mathrm{j}}+\mathrm{FMs}_{\mathrm{ij}}+$ $R_{k}+\varepsilon_{i j k} ; Y_{i j k}$ is the observed value for the cross between the ith and jth parents in the kth replication; $\mu$ is the population mean; $\mathrm{Fg}_{\mathrm{i}}$ is the GCA value of the ith female parent; $\mathrm{Mg}_{\mathrm{j}}$ is the GCA value of the jth male parent; $\mathrm{FMs}_{\mathrm{ij}}$ is the SCA value for the cross between the ith and $j$ th parent; $R_{k}$ is the replication effect; $\varepsilon_{i j k}$ is the error associated with each observation.

\section{Conclusion}

In conclusion, having produced progenies with resistance to CMD, there is a need to conduct extensive GXE evaluation of the stability of the resistance in association with expression of the important agronomic traits. The adoption of appropriate strategies to exploit the additive and non-additive gene action determining CMD resistance and the other traits is now possible. The prospects for substantial improvements in the productivity of cassava cultivars grown in Zambia are very real and exciting indeed.

\section{Acknowledgement}

We acknowledge Agriculture Green Revolution in Africa (AGRA) for the financial assistance in carrying out the study. The authors gratefully thank Mr. Mathias Tembo and Ms. Dina Mambwe for grafting and data collection, respectively.

\section{References}

Angarawai II, Kadams AM, Bello D (2008) Gene effects controlling heritability of downy mildew resistance in Nigerian elite pearl millet lines. World J Agri Sci. 4:545549.

Cach NT, Lenis JI, Perez JC, Morante N, Calle F, Ceballos $\mathrm{H}$ (2006) Inheritance of useful traits in cassava grown in sub-humid conditions. Plant Breeding. 125:177-182.

Calvert LA, Thresh JM (2002) The viruses and virus diseases of cassava. In:, Hillocks et al (eds) Biology, production and utilisation. CABI Publishing, New York.

Ceballos H, Iglesias CA, Perez JC, Dixon AGO (2004) Cassava breeding: opportunities and challenges. Plant Mol Biol. 56:503-516.

Eberhart SA, Gardner CO (1966) A general model for genetic effects. Biometrics. 22:864-881.

Hahn SK, Terry ER, Leuschner K (1980) Breeding cassava for resistance to cassava mosaic disease. Euphytica. 29:673-683.

Hahn SK, Terry ER, Leuschner K, Akobundu IO, Okali C, and Lal R (1979) Cassava improvement in Africa. Field Crops Res. 2:192-226.

Hallauer AR, Miranda JB (1988) Quantitative genetics in maize breeding. Second ed Iowa State University Press, Iowa.

Haussmann BIG, Obilana AB, Blum AP, Ayiecho O, Schipprack W, Geiger HH (1999) Quantitative-genetic parameters of sorghum (Sorghum bicolor (L) Moench) 
grown in semi-arid areas of Kenya. Euphytica 105:109118

Hogarth DM, Wu KK, Heinz DJ (1981) Estimating genetic variance in sugarcane using a factorial cross design. Crop Sci. 21:21-25.

Jennings DL, Iglesias CA (2002) Breeding for crop improvement. In: Hillock et al (eds) Cassava: Biology, Production and Utilisation. CABI Publishing, New York.

Jollife IT (2002) Principal component analysis. 2nd ed Springer-Verlag New York, Inc USA.

Kamau J, Melis R, Laing M, Derera J, Shanahan P, Ngugi E (2010) Combining the yield ability and secondary traits of selected cassava genotypes in the semi-arid areas of Eastern Kenya. J Plant Breed Crop Sci. 2:181-191.

Kawano K (2003) Thirty years of cassava breeding for productivity: Biological and social factors for success. Crop Sci. 43:1325-1335.

Kawano K, Narintaraporn K, Narintaraporn P, Sarakarn S, Limsila A, Limsila J, Suparhan D, Sarawat V, Watananonta W (1998) Yield improvement in a multistage breeding programme for cassava. Crop Sci. 38:325-332.

Legg JP, Jeremiah SC, Obiero HM, Maruthi MN, Ndyetabula I, Okao-Okuja G, Bouwmeester H, Bigirimana S, TataHangy W, Gashaka G, Mkamilo G, Alicai T, Lava Kumar $P$ (2011) Comparing the regional epidemiology of the cassava mosaic and cassava brown streak virus pandemics in Africa. Virus Res.159(2):161-70.

Lenis JI, Calle F, Jaramillo G, Perez JC, Ceballos H, Cock JH (2006) Leaf retention and cassava productivity. Field Crops Res. 95:126-134.

Lokko Y, Dixon AGO, Offei SK, Danquah EY (2006) Combining ability analysis of resistance to mosaic virus disease in cassava. Afr Crop Sci J. 14:221-230.

Mtunda KJ (2010) Breeding, Evaluation and Selection of Cassava for High Starch Content and Yield in Tanzania. $\mathrm{PhD}$ thesis University of KwaZulu-Natal, South Africa.

Muimba-Kankolongo A, Chalwe A, Sisupo P, Kang MS (1997) Distribution, prevalence and outlook for control of cassava mosaic disease in Zambia. Roots. 4:2-7.

Munga TL (2008) Breeding for cassava brown streak resistance in coastal Kenya. PhD Thesis University of KwaZulu-Natal, South Africa.
Ngeve MJ (1999) Agronomic performances, yield stability and field disease reaction of cassava genotypes in the subhumid forest region of Cameroon. Afr Crop Sci J. 7:129142.

Ragsdale PI, Smith W (2007) Germplasm potential for trait improvement in upland cotton: diallel analysis of within boll seed yield components. Crop Sci. 47:1013-1017.

Thresh JM, Cooter RJ (2005) Strategies for controlling cassava mosaic virus disease in Africa. Plant Pathol. 54:587-614.

Thresh JM, Fargette D, Otim-Nape GW (1994) Effects of African cassava mosaic geminivirus on the yield of cassava. Tropical Sci. 34:26-42.

Virk DS, Khehra AS, Virk PS, Dhillon BS (1985) Comparative genetic analysis of metric traits using diallel and factorial mating designs in bread wheat. Theorl Appl Genet. 69:325-328.

Zacarias AM (2008) Breeding potential of cassava (Manihot esculenta Crantz) in Mozambique. PhD thesis, University of the Free State, South Africa. 